\title{
Fluctuation Induced First Order Phase Transitions
}

\author{
Yue Shen ${ }^{\mathrm{a} *}$ \\ aPhysics Department, Boston University, Boston, MA 02115, USA
}

We study a $U(N) \times U(N)$ symmetric scalar field model in four and three dimensions. First, using our data in four dimensions in the weak coupling region, we demonstrate explicitly that the observed first order phase transition is induced by quantum fluctuations. Next, based on the renormalization group and our new simulation results in three dimensions we argue that even if the $U_{A}(1)$ symmetry is restored below the critical temperature the QCD finite temperature chiral phase transition for two flavor could be extremely weak first order.

\section{The Model}

Let us consider a scalar field model with action

$$
\begin{aligned}
S_{\phi} & =\int d^{D} x\left\{\frac{1}{2} \operatorname{tr}\left(\partial_{\mu} \phi^{\dagger} \partial_{\mu} \phi\right)+\frac{m^{2}}{2} \operatorname{tr}\left(\phi^{\dagger} \phi\right)\right. \\
& \left.+\lambda_{1}\left(\operatorname{tr} \phi^{\dagger} \phi\right)^{2}+\lambda_{2} \operatorname{tr}\left(\phi^{\dagger} \phi\right)^{2}\right\}
\end{aligned}
$$

where $\phi$ is a $N \times N$ complex matrix and $D$ is space-time dimension. It has $U(N) \times U(N)$ global symmetry under transformation: $\phi \rightarrow U \phi V^{\dagger}$, where $U$ and $V$ are $N \times N$ unitary matrices.

In contrast to a $O(N)$ scalar model, the symmetry breaking phase transition (PT) for Eq. (11) can be first order due to the well known ColemanWeinberg phenomenon [1]. A qualitative argument for the Coleman-Weinberg phenomenon has been developed based on the effective potential and the renormalization group (RG) [1]. Essentially first order PT occurs due to the existence of run-away RG trajectories. However, physical applications of Eq. (1) rely on the RG flow obtained in perturbation [2] or $D=4-\epsilon$ expansion extrapolated to $\epsilon=1[3]$. It is important to extend these arguments in a nonperturbative study.

\section{2. $\mathrm{D}=4$}

Our first numerical results in 4D are reported in Ref. [4], which we refer to for details of the lattice simulations and discussions of their relevance to

\footnotetext{
*This work was supported in part under DOE contract DE-FG02-91ER40676 and NSF contract PHY-9057173, and by funds from the Texas National Research Laboratory Commission under grant RGFY92B6.
}

the continuum physics. As an illustration for the quantum fluctuation induced first order PT, we plot in Fig. 1 a comparison of the one-loop perturbative calculation with MC data in the weak coupling region. Here the one-loop effect changes the second order PT at the tree level to first order. First order PT were also found in the strong coupling region [4], indicating the absence of a stable infrared fixed point there.

\section{3. $\mathrm{D}=3$}

In three dimensions, Eq. (11) can be used to describe the QCD finite temperature chiral phase transition (ChPT) [3]. Here the order of PT depends crucially on the number of fermion flavor $N_{f}$ and the pattern of symmetry. For $N_{f} \geq 3 \mathrm{ChPT}$ is in general expected to be first order [3]. For $N_{f}=2$, if the axial $U_{A}(1)$ anomaly remains effective up to the ChPT temperature $T_{c h}$, the symmetry of the system will be $S U(2) \times S U(2) \sim O(4)$ and the transition can be second order. However, $U_{A}(1)$ symmetry could be restored below $T_{c h}$, the symmetry becomes $U_{A}(1) \times S U(2) \times S U(2)$ and $\mathrm{ChPT}$ would be first order 3].

So far there is no numerical evidence for a first order ChPT for $N_{f}=2$ [5]. Does this imply that the $U_{A}(1)$ symmetry is not restored below $T_{c h}$ ? The answer can not be conclusive. In fact, as we will show in the following using the effective action in Eq. (1): even if we assume the $U_{A}(1)$ symmetry is restored below $T_{c h}$, ChPT can be very weakly first order. 


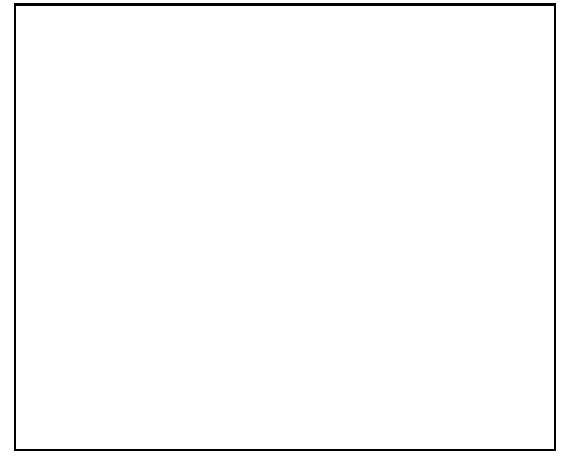

Figure 1. Comparison of the numerical results with the bare perturbation calculation (dotted line) in 4D. The numerical data are obtained on the $4^{4}$ (open circle), $6^{4}$ (open square) and $10^{4}$ (open triangle) lattices. The solid lines connect $6^{4}$ and $10^{4}$ data points to indicate the hysteresis effects.

For $N=2$ the RG flow for Eq. (11) is plotted in Fig. 2 where the $\beta$-functions are obtained in $D=4-\epsilon$ expansion with $\epsilon=1$. There is a UV fixed point at $\lambda_{1}=\lambda_{2}=0$ (solid circle) and a unstable IR fixed point at $\lambda_{1}=\pi^{2} \epsilon / 8, \lambda_{2}=0$ (solid square). The dotted lines in Fig. 2 indicate the "stability line" (SL). Qualitatively, for a renormalized theory located to the left side of SL, PT will be first order [1]. However, PT can be weakly or strongly first order depending on the choice of the bare Lagrangian. The RG running of $\lambda_{1}, \lambda_{2}$ is very slow in the weak coupling region and becomes fast in the strong $\lambda_{2}$ region. A weak coupling bare action chosen to the right side of SL will need many decades of running in energy scale in order to cross SL. Therefore, even though the PT will be first order, the jump in the vacuum expectation value $(\mathrm{VEV}), v$, at the transition point can be many orders of magnitude smaller than the "cut-off" scale $\Lambda$ and the transition can be very weakly first order [4].

As an example, we plot $2 v^{2}=\left\langle\operatorname{tr} \bar{\phi}^{\dagger} \bar{\phi}\right\rangle$ in Fig. 3 for $\lambda_{1}=\lambda_{2}=0.0625$. Obviously, it is almost impossible to decide numerically at this point if the $\mathrm{PT}$ is second order or weakly first order. Here we

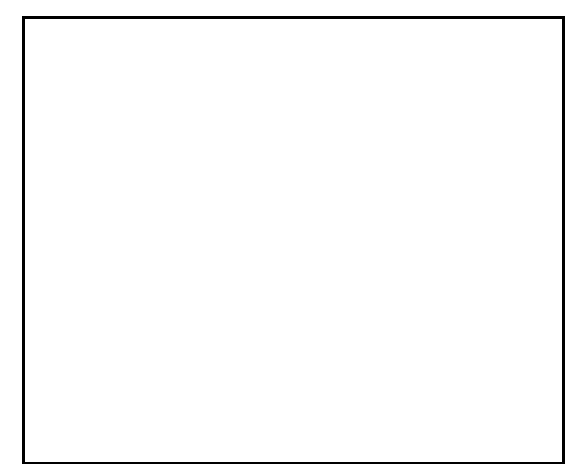

Figure 2. RG flow in 3D. The arrows point to the infrared direction.

wish to correct an error appeared in the literature [6]. The point at $m^{2}=-6.8$ (solid circle in Fig. 3) which was claimed to be the critical point of a first order PT [6] is actually deeply in the broken phase.

The search for a first order PT is further complicated on a finite lattice. The running of $\lambda_{1}, \lambda_{2}$ is controlled by the correlation length $\xi$, which is limited by the box size $\xi \leq L$. Therefore, a bare action fixed to the right of SL will only be able to run across SL in a large enough box [4]. The first order nature of the PT will not appear until the simulation is performed on a large lattice. There is an interesting example of this phenomenon in the literature [7]. In the simulation of $U(N) \times U(N)$ nonlinear sigma model, which is in the same universality class as Eq. (1), it was found that for $N=2$ the first order nature of the PT could be exposed only on lattices larger than $14^{3}$. We should also point out that for the same initial values of $\lambda_{1}, \lambda_{2}$, the RG running is faster with increasing $N$. This explains the stronger first order PT for $N=3$ in Ref. [7].

To observe the first order PT in a small box, one either has to move the bare action close to SL in the weak coupling region, or move to the strong $\lambda_{2}$ region where the speed of running is fast [4]. We give an example in Fig. 4 where the bare action is set to be very close to SL. We are able to observe clear signal for a first order PT 


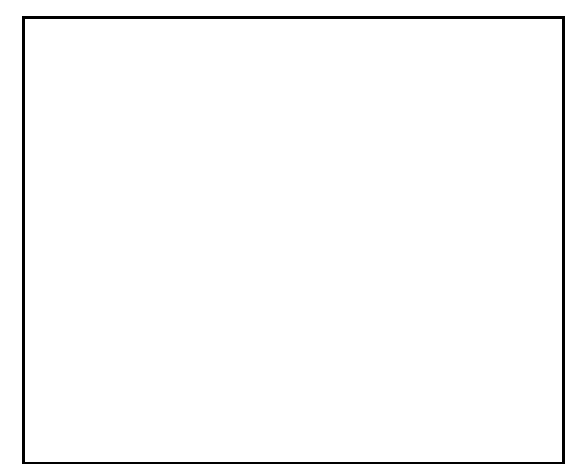

Figure 3. In 3D, $\left\langle\operatorname{tr}\left(\bar{\phi}^{\dagger} \bar{\phi}\right)\right\rangle$ as a function of $m^{2}$ at $\lambda_{1}=\lambda_{2}=0.0625$. The bare perturbation prediction is given by the solid line.

on the $6^{3}$ lattice.

What are the implications for finite temperature QCD? Let us assume that Eq. (1) becomes a good effective theory for QCD at energy scale $\Lambda<2 \pi \Lambda_{Q C D}$. Then the strength of first order PT crucially depends on the values of the effective coupling at scale $\Lambda$. As discussed above, if $\lambda_{1}(\Lambda), \lambda_{2}(\Lambda)$ fall into the weak coupling region, finding the first order PT will become a tough job. While it is not possible to determine $\lambda_{1}(\Lambda), \lambda_{2}(\Lambda)$ for QCD, it was found in a large color $\left(N_{c}\right)$ approximation for the Nambu-Jona-Lasinio (NJL) model that $\lambda_{1}=0, \lambda_{2}=2 \pi^{2} / N_{c} \ln \left(\Lambda_{N J L} / \Lambda\right)$ ] where $\Lambda_{N J L}$ is the cut-off for NJL model and $\Lambda$ is the scale when the linear sigma model becomes a good effective theory. Although NJL model is a poor approximation of QCD, we might believe that it is at least qualitatively correct. Then $\lambda_{2}$ can be strong or weak depending on the ratio $\Lambda_{N J L} / \Lambda$ (one might take $\Lambda_{N J L} \sim 4 \pi \Lambda_{Q C D}$ ). Since $\lambda_{2}$ does not depend on $N_{f}$, the only feature that distinguishes flavor is the speed of RG flow. We could immediately draw a conclusion that it would be easier to observe the first order PT for $N_{f}=4$ than $N_{f}=2$, which agrees with well known simulation results [5]. Even in the case of $4 \pi \Lambda_{Q C D} / \Lambda \sim 1$ and $\lambda_{2}$ is very large (Eq. (1) becomes effectively the nonlinear sigma model), results of Ref. [7] indicate that one may need a

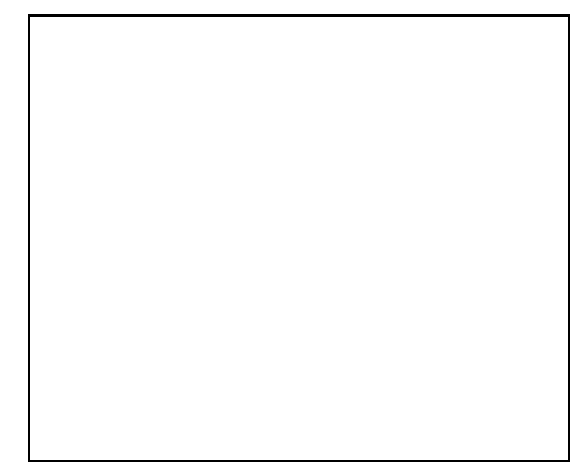

Figure 4. In 3D, Monte Carlo time history for $\operatorname{tr}\left(\bar{\phi}^{\dagger} \bar{\phi}\right)$ at $\lambda_{1}=-0.22, \lambda_{2}=0.5, m^{2}=-1.038$. Because of the closeness to the "stability line", we are able to observe tunneling between two states on a $6^{3}$ lattice.

quite large lattice to expose the first order nature of PT.

Beyond large $N_{c}$ limit, one in principle can not eliminate the possibility of a stronger $N_{f}$ dependence for QCD. Then for $N_{f}=2, \lambda_{2}(\Lambda)$ could fall in the very weak coupling region such that $\mathrm{PT}$ is extremely weak first order.

I thank R. Pisarski for useful discussions.

\section{REFERENCES}

1. For review, see, D. J. Amit, Field Theory, the Renormalization Group and Critical Phenomena, 2nd ed., World Scientific, Singapore, 1984.

2. R. S. Chivukula, M. Golden and E. Simmons, Phys. Rev. Lett. 70 (1993) 1587.

3. R. D. Pisarski and F. Wilczek, Phys. Rev. D29 (1984) 338.

4. Y. Shen, Phys. Lett. B315 (1993) 146.

5. F. Karsch, these proceedings.

6. H. Gausterer and S. Sanielevici, Phys. Lett. B209 (1988) 533.

7. P. Dreher, Nucl. Phys. (Proc. Suppl.) 9 (1989) 646; Nucl. Phys. (Proc. Suppl.) 20 (1991) 288; Phys. Lett. B281 (1992) 127.

8. W. A. Bardeen, C. T. Hill and D. U. Jungnickel, preprint SSCL-Preprint-490. 
This figure "fig1-1.png" is available in "png" format from: http://arxiv.org/ps/hep-lat/9311018v1 
Figure 1

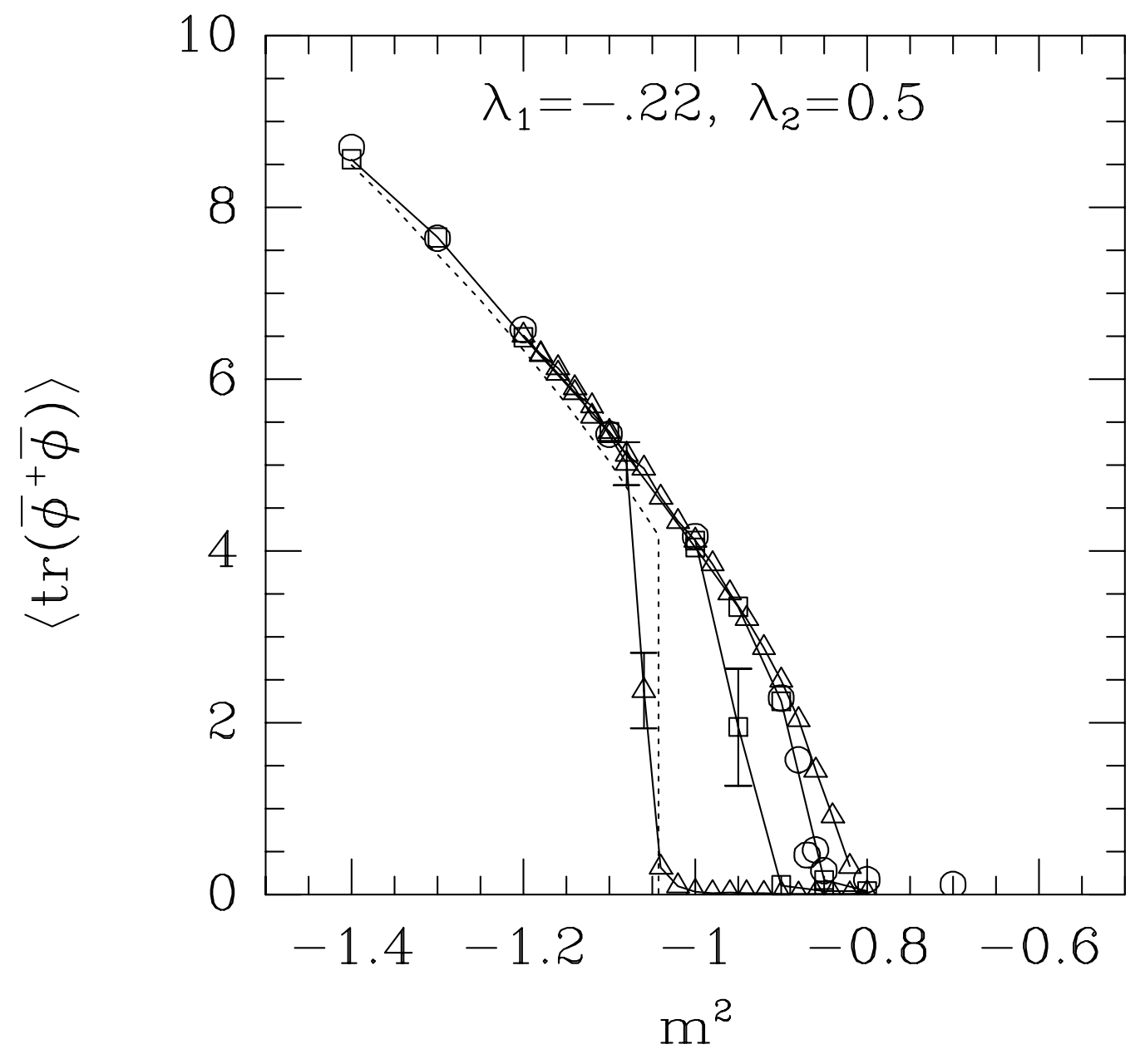


This figure "fig2-1.png" is available in "png" format from: http://arxiv.org/ps/hep-lat/9311018v1 
This figure "fig1-2.png" is available in "png" format from: http://arxiv.org/ps/hep-lat/9311018v1 
This figure "fig2-2.png" is available in "png" format from: http://arxiv.org/ps/hep-lat/9311018v1 
Figure 2

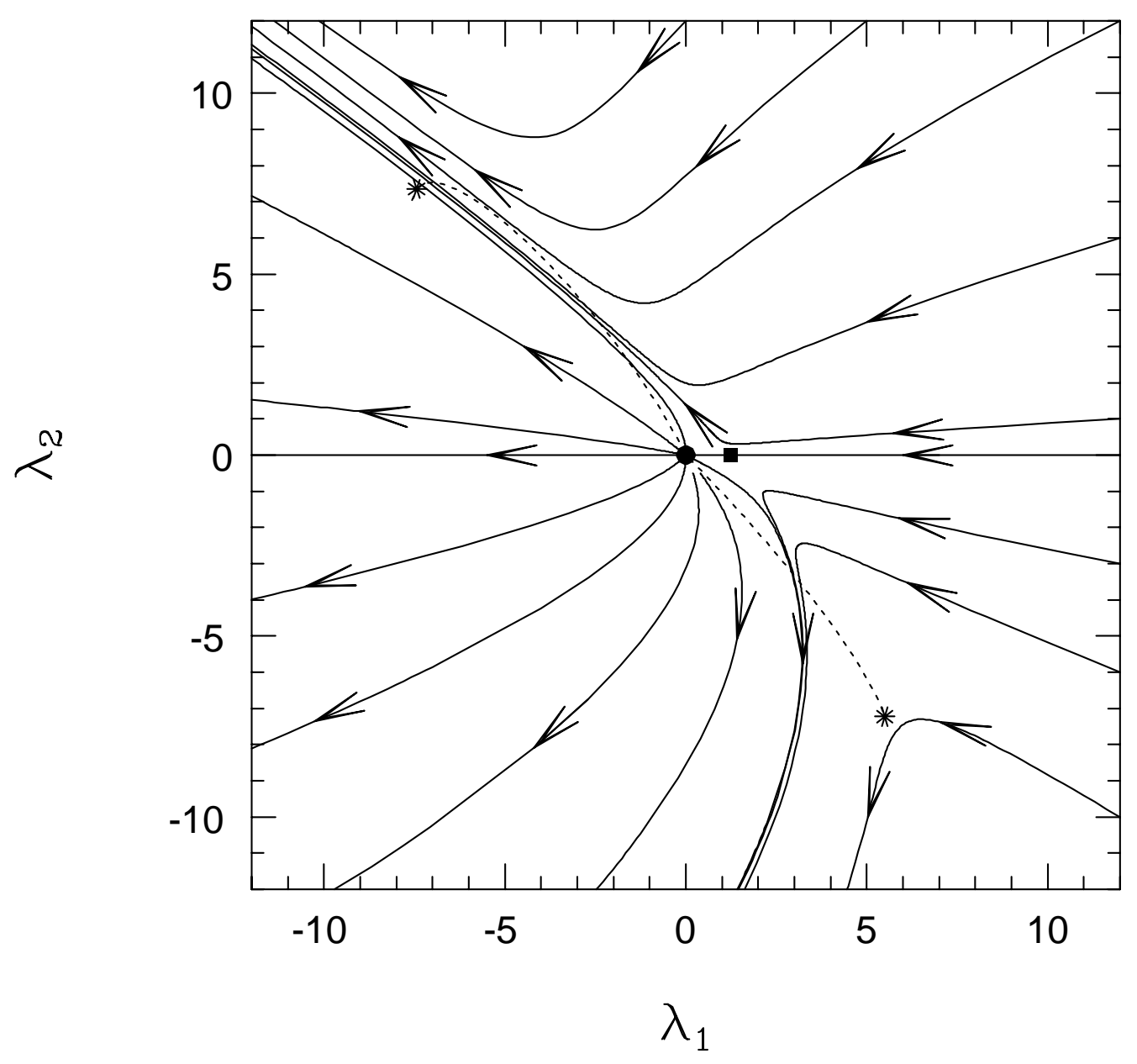


Figure 3

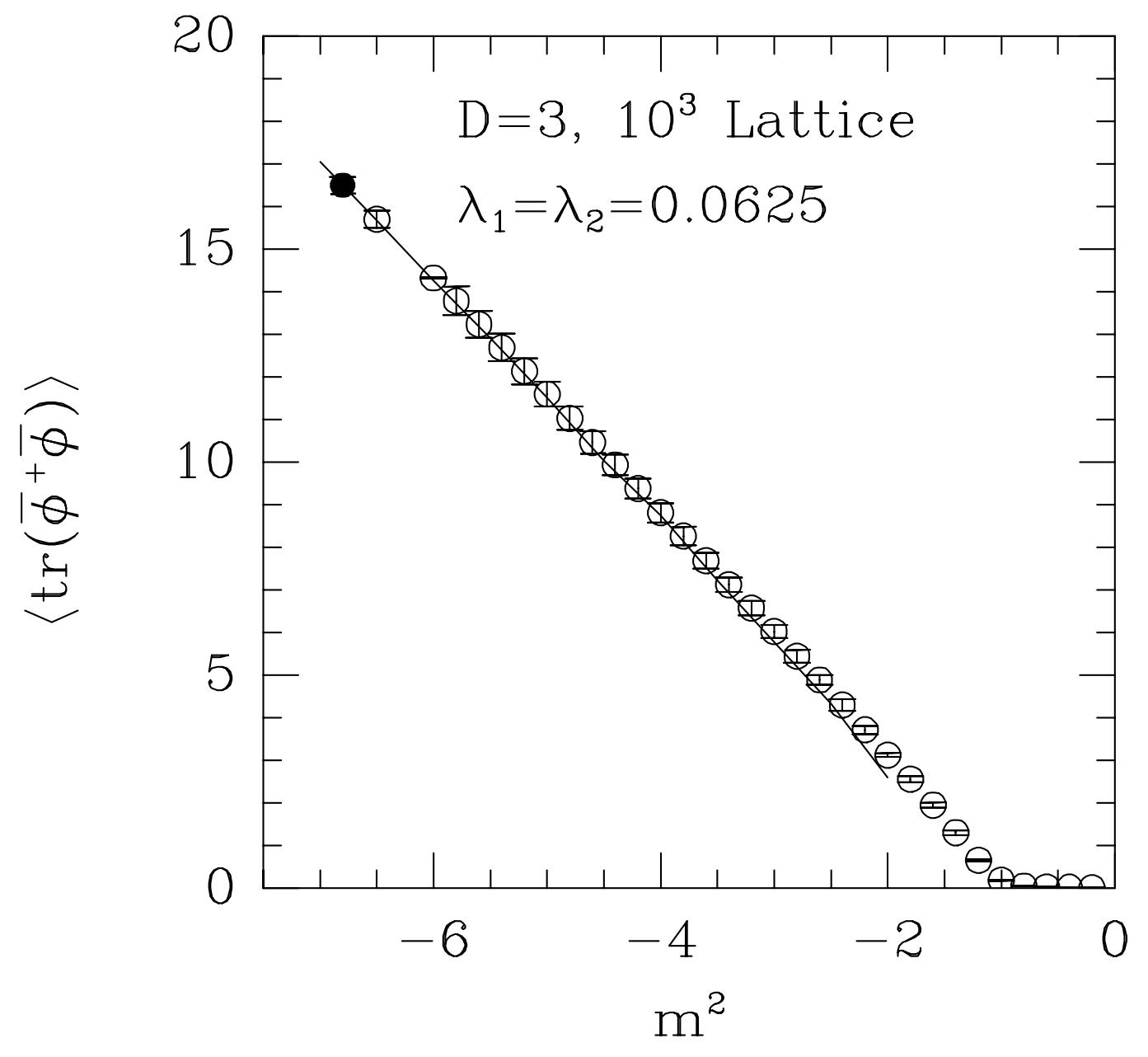


Figure 4

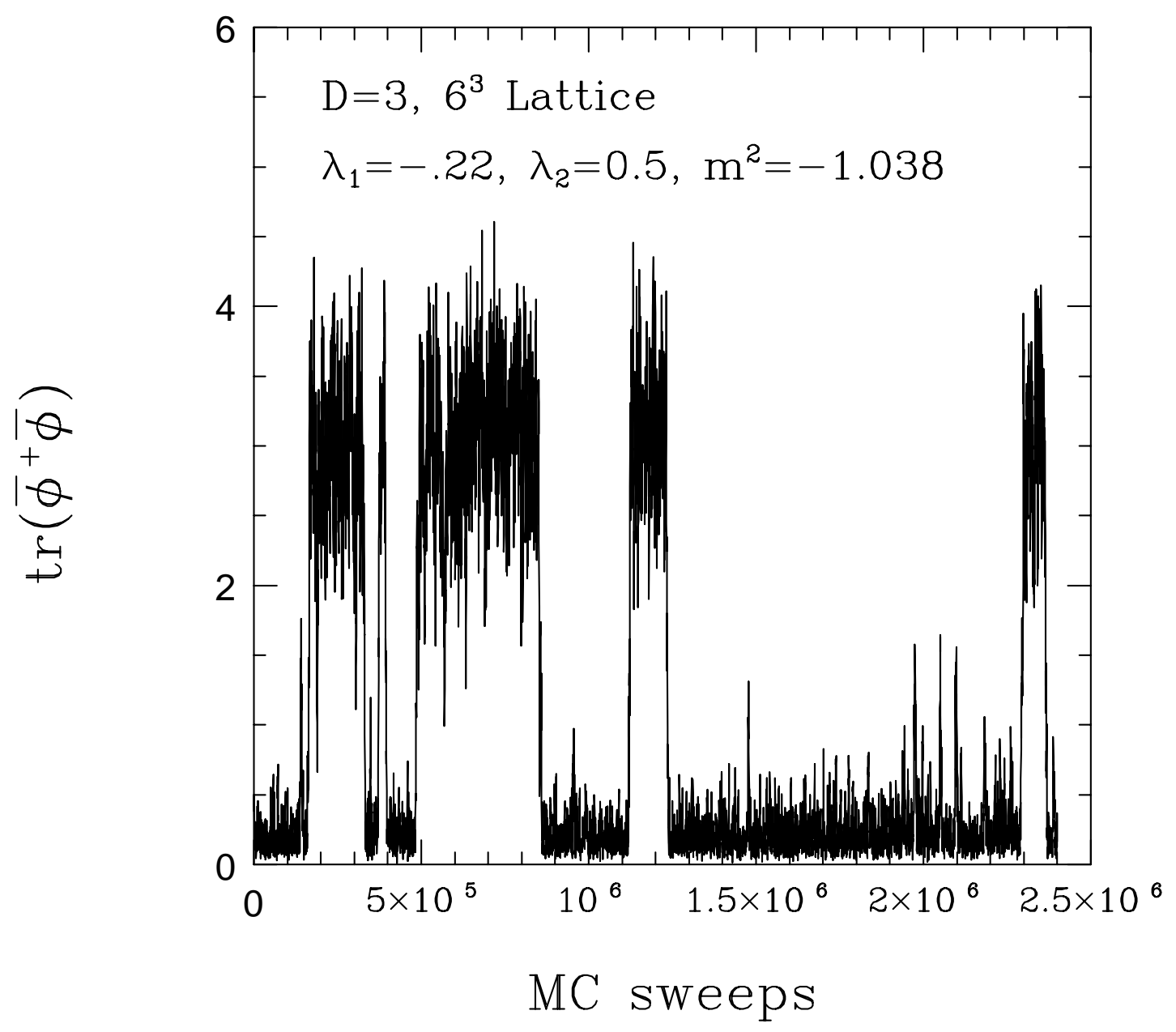

